\title{
Bayesian Logistic Regression of Soybean Sclerotinia Stem Rot Prevalence in the U.S. North-Central Region: Accounting for Uncertainty in Parameter Estimation
}

\author{
A. L. Mila, X. B. Yang, and A. L. Carriquiry
}

First and second authors: Department of Plant Pathology; and third author: Department of Statistics, Iowa State University, Ames 50011. Current address of A. L. Mila: Department of Plant Pathology, University of California-Davis, Kearney Agricultural Center, Parlier 93648. Accepted for publication 27 December 2002.

\section{ABSTRACT}

Mila, A. L., Yang, X. B., and Carriquiry, A. L. 2003. Bayesian logistic regression of soybean Sclerotinia stem rot prevalence in the U.S. northcentral region: Accounting for uncertainty in parameter estimation. Phytopathology 93:758-764.

Bayesian ideas have recently gained considerable ground in several scientific fields mainly due to the rapid progress in computing resources. Nevertheless, in plant epidemiology, Bayesian methodology is not yet commonly discussed or applied. Results of a logistic regression analysis of a 4-year data set collected between 1995 and 1998 on soybean Scle- rotinia stem rot (SSR) prevalence in the north-central region of the United States were reexamined with Bayesian methodology. The objective of this study was to use Bayesian methodology to explore the level of uncertainty associated with the parameter estimates derived from the logistic regression analysis of SSR prevalence. Our results suggest that the 4-year data set used in the logistic regression analysis of SSR prevalence in the north-central region of the United States may not be informative enough to produce reliable estimates of the effect of some explanatory variables on SSR prevalence. Such confident estimations are necessary for deriving robust conclusions and high quality predictions.
Plant pathologists face a variety of challenges every time they analyze their experimental results, fit probability models in complex data sets, draw conclusions about the present and make predictions for the future. Currently, most statistical analyses are performed with the help of commercial software packages, most of which use methods based on the framework of classical statistical philosophy. In this framework, maximum likelihood estimates (MLEs) and hypothesis tests based on $P$ values figure prominently.

The idea behind maximum likelihood parameter estimation is to determine the parameter values that maximize the probability (likelihood) of the sample data, i.e., values that are most consistent with the sample data. In general, the sample size is associated to the accuracy of an estimator. If the sample equals the entire population, then the estimator is the true value. From a statistical point of view, the method of maximum likelihood yields estimators with good statistical properties. Uncertainty associated with parameter estimation is quantified through confidence bounds that are often established using large sample (asymptotic) arguments.

Bayesian parameter estimation methods, in contrast, rely not only on current knowledge (sample data) but also on prior information that may be available on the parameters of interest. Thus, an important difference between the classical and the Bayesian framework is the introduction of prior information in the form of probability distributions (4). Moreover, in the Bayesian framework conclusions about parameters are made in terms of a probability statement, i.e., parameter estimates are no longer expressed as point estimates but instead are statistical distributions (4). Uncertainty associated to parameter estimation is quantified through the use of these probability distributions (7).

Corresponding author: A. L. Mila; E-mail address: mmila@uckac.edu

Publication no. P-2003-0407-010

(C) 2003 The American Phytopathological Society
In a previous study (12), regional prevalence of soybean Sclerotinia stem rot (SSR), caused by Sclerotinia sclerotiorum, was analyzed using a logistic regression model. Estimation of the probability of stem rot prevalence was made with disease data from four states (Illinois, Iowa, Minnesota, and Ohio) in the northcentral region of the United States. Tillage practices, average air temperature of July and August, and precipitation in July and August were used as input variables. Potential differences between states in SSR prevalence were addressed by including regional indicator variables in the regression model. Because the parameter estimates were made with the classical approach, it would be interesting to examine the level of uncertainties associated with these estimates using Bayesian methodology.

Although scientists do not typically focus on assessing uncertainties associated with parameter estimation or model prediction, these are always present. Thus, one challenge is to accurately identify those variables in the model for which the sample data do not provide enough information for reliable estimation and to investigate the possibility of improving estimation through the incorporation of any available prior information. The objective of the present study was to evaluate the level of uncertainty associated with the parameter estimates derived from the logistic regression analysis of SSR prevalence in the north-central region of the United States using data collected between 1995 and 1998 (12). We aimed at investigating if the four-year data set was informative enough so that the derived estimates for the explanatory variables of SSR prevalence were reliable.

\section{MATERIALS AND METHODS}

Concepts of Bayesian analysis. In the Bayesian framework, there are three key components associated with parameter estimation: the prior distribution, the likelihood function, and the pos- 
terior distribution. These three components are formally combined Bayes' rule by

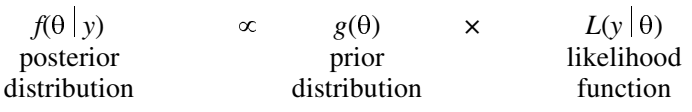

where the symbol $\propto$ denotes proportionality (7). In simple terms, equation 1 states that the information contained in the sample (reflected in the likelihood function) is combined with information from other sources (summarized by the prior distribution). The posterior distribution contains all the available knowledge about the parameters in the model.

Existing evidence about the parameters of interest may be available from earlier studies or from experts' opinions, and can be formalized into what is called the prior distribution of the parameter of interest (Fig. 1). A prior distribution can be noninformative, informative, or very informative. Noninformative prior distributions are used in cases in which no extra-sample information is available on the value of the parameters of interest. In statistical terms, this lack of knowledge is represented with a distribution that attributes approximately the same probability to each possible parameter value (Fig. 1A). Informative prior distributions are used when some prior knowledge about the parameters of interest is available, such as when existing belief or evidence indicates that a parameter should take a value within a range (6). Formally, this knowledge is represented with a distribution that has a known mean and large variance (Fig. 1B). Very informative prior distributions are used when very strong prior knowledge about the parameters of interest is available, such as when existing belief or evidence indicates that a parameter of interest should be a specific value. In statistical terms, this knowledge can be represented with a distribution that has a known mean and small variance (Fig. 1C). The choice of an informative prior distribution typically involves a certain amount of subjectivity; historically, this has been a reason for disagreement between Bayesian and classical statisticians.

Data collected in the course of the present experiment are used to form the likelihood function. The relative contributions of the likelihood function and the prior distribution to the posterior depend on the sample size; when the sample size is very large, the data dominate the prior distribution with parameter estimates derived mainly from the information residing in the sample. It is important to notice that the likelihood function used in Bayesian analysis also forms the basis of classical statistical analysis (7). Thus, the main differences between classical and Bayesian models are the introduction of the prior distributions and the interpretation of the results.

SSR prevalence study. Likelihood function. In our SSR prevalence study, the likelihood function is based on the data set obtained through a survey conducted from 1995 to 1998 in Illinois, Iowa, Minnesota, and Ohio. In total, 1,853 fields were randomly sampled during the 4-year period. From each selected field, 20 soybean stems were sampled in a zigzag pattern and shipped to Iowa State University for examination. Survey methods have been previously described (16).

Prior distributions. For comparison, two sets of prior distributions for the model parameters were examined: noninformative and very informative. The hierarchical model structure is explained in the appendix.

Explanatory variables. Explanatory variables used in the present study were selected based on a previous logistic regression analysis (12). These were average air temperature of July and August, total precipitation of July and August, an indicator variable for tillage effect (with conventional tillage arbitrarily used as the reference group), and an indicator variable to represent state effect (with Illinois arbitrarily used as the reference group).

Assumptions used to construct very informative prior distributions. The following assumptions were made for defining the very informative prior parameter distributions. (i) The regression coefficient associated to average air temperature of July and August should be negative. This assumption is based on previous literature $(1,3,8)$ that Sclerotinia stem rot is a cool temperature disease. (ii) The regression coefficient associated to July and August precipitation should be positive. Several epidemiological studies $(1,3,13,15)$ demonstrate the importance of precipitation for Sclerotinia rot occurrence because prolonged periods of high soil moisture are favorable for apothecial development (2). (iii) Previous studies $(1,3,13,14)$ show that precipitation around time of flowering is a main limiting factor for the disease development. Given this epidemiological aspect of Sclerotinia diseases that seems to be similar in more than one crop, it was remarkable that the regression parameter estimate from logistic regression associated to July precipitation was $1 / 10$ of the parameter estimate for the average temperature of July and August. Further, it was rather unexpected that the August precipitation parameter estimate was not significant at the $5 \%$ level of significance (Table 1). The above estimations indicate that temperature, and not precipitation, is the most important factor for soybean SSR prevalence in the northcentral region of the United States. However, because only 4 years of data were used in the logistic regression analysis, it is questionable whether these data provide a reliable estimate of the effect of precipitation on the SSR prevalence. To address this question, very informative prior distributions were imposed on the July and August precipitation effect; we assumed that the July precipitation effect was equal to the temperature effect (i.e., mean of prior distribution equal to 0.4) and the August precipitation effect was half of the July precipitation effect (i.e., mean of prior distribution equal to 0.2). (iv) Based on previous studies $(5,10)$, minimum till-

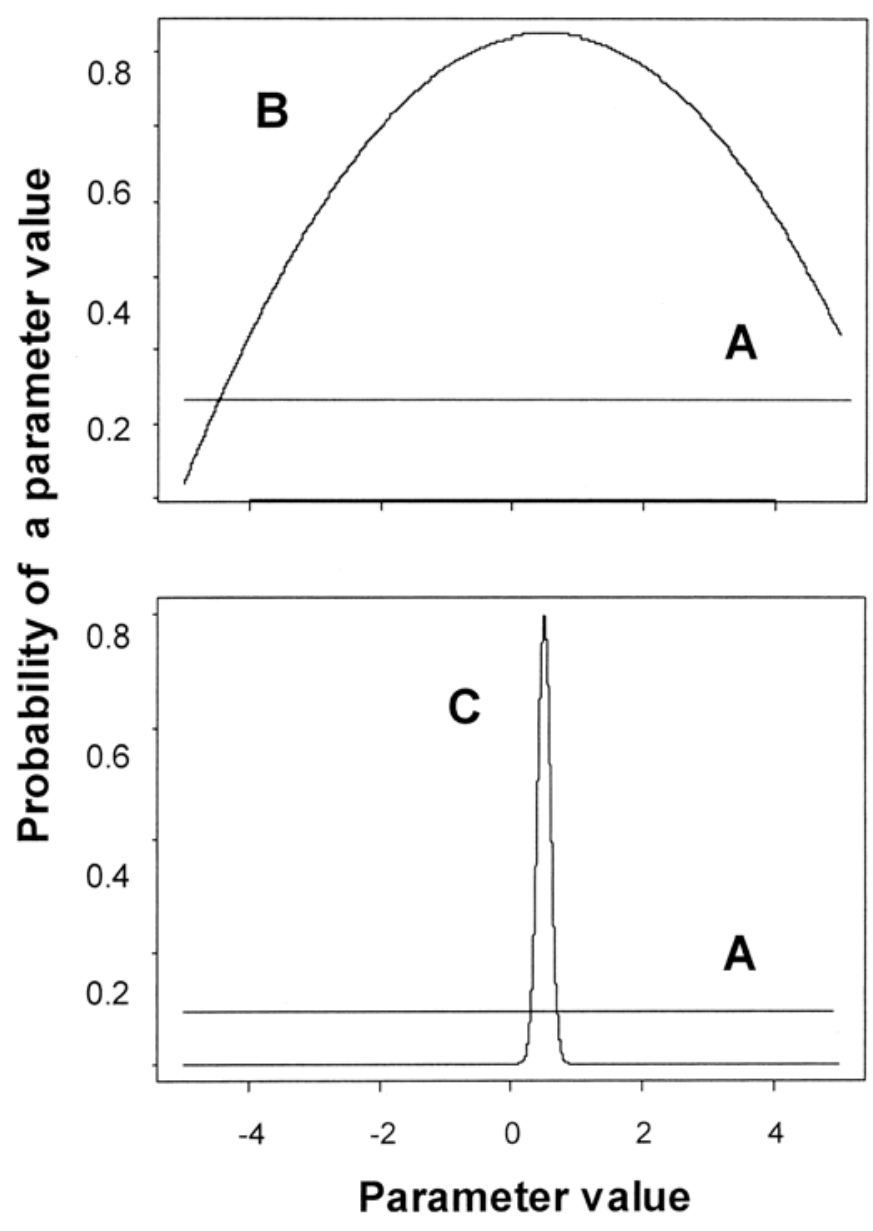

Fig. 1. Illustrations of $\mathbf{A}$, noninformative, $\mathbf{B}$, informative, and $\mathbf{C}$, very informative parameter prior distributions in Bayesian analysis. 
age was the system with the highest risk and no tillage was the system with the lowest risk, and (vi) from the four states used in the analysis, Minnesota was considered to have the highest risk and Ohio the lowest risk. To form the very informative prior distribution for the state effect, we used a technique called Empirical Bayes, i.e., we formed our prior distributions using information from the sample. The reason for using this technique was that there was no previous survey on SSR in the north-central region to provide prior information on the relative risk of the disease in each state.

It should be clarified that the use of informative prior distributions was primarily to examine the degree of uncertainty associated with the parameter estimates. If the purpose is to improve the parameter estimations, detailed and precise information on the effect of each factor on SSR prevalence is needed.

Statistical analysis. Estimation of the posterior distributions of the parameters was carried out using the Gibbs sampler (GS). GS is an iterative algorithm that produces a Markov chain and permits empirical estimation of posterior distributions. The analysis was implemented with BUGS (Bayesian Using Gibbs sampling) software (WinBugs, version 1.3, MRC, Biostatistics Unit, Cambridge, UK). CODA, a suite of S-plus (Mathsoft Inc., Cambridge, MA) functions, was used for plotting the BUGS output files and for diagnosing convergence of the algorithm. All other calculations were performed with S-plus.

Monitoring convergence of GS is a very important step in Bayesian analysis because only the iterations after convergence are used to obtain estimates of parameter distributions (such as mean, median, standard deviation, and quantiles). For monitoring convergence, it is often recommended that several chains are generated independently for each model parameter (6). Visual examination of trace plots, autocorrelations, and Gelman-Rubin (GR) diagnostic were applied to diagnose convergence. GR uses several parallel chains with widely dispersed starting values with respect to the true posterior distribution to assess convergence. This statistic compares the variability between and within chains by estimating a potential scale reduction factor. Approximate convergence is diagnosed when the variance between the different chains is no larger than the variance within each individual chain and their ratio is approximately equal to one (6). Based on the convergence criteria mentioned previously, we generated three parallel runs of 10,000 iterations with dispersed starting values for each model parameter. Posterior inference was based on empirical summaries of the final 2,000 samples in each run.

Because Bayesian analysis results in distributions (called posterior distributions) for each parameter in the model, parameter estimates do not consist of just a point estimate as is the usual case, but instead consist of entire distributions that can be summarized via, for example, medians and quantiles (4). These quantiles can be useful for comparing distributions and for assessing changes that may occur under different modeling assumptions. Another commonly used summary is the probability that a parameter is below or above zero, and can be used instead of the $P$ value, which is used in classical statistics to determine whether a parameter is statistically significant or not.

\section{RESULTS}

Convergence. For all parameters, the trace plots of the last 200 iterations for three independently generated chains demonstrated good "chain mixture" (an example is in Fig. 2A), an indication of convergence. Autocorrelation values across successive parameter draws ranged from 0.05 to 0.25 , indicating that the realized value of a parameter in a given iteration did not depend on the sampled values in the preceding iterations (Fig. 2B). When the autocorrelation across sampled parameter values is low, the final sample used for estimation is closer to a random sample. GR also indicated that there was convergence of the chains (Fig. 2C, line 3 approximately equal to 1). The posterior distributions of the model parameters obtained from the sampled values reflected smooth kernel densities (Fig. 3A to D).

Noninformative prior distributions. Posterior point parameter estimates (posterior means) were approximately equal to the estimates generated with logistic regression analysis (12) (Table 1). However, elements such as the quantiles of the parameter posterior distributions (Table 1) and the posterior probability of positive or negative values for each input parameter (Table 2) are of primary interest. For example, the estimated posterior mean of the effect of the average temperature of July and August is -0.47 with $(-0.678,-0.268)$ as a $95 \%$ credible interval. Similar results would also arise from a classical statistical analysis. However, the parameter for July and August average temperature is a distribution (Fig. 3A) and further conclusions can be derived. For example, there is a small chance $(2.5 \%)$ to have an estimate of the very low value of -0.678 or the high value of -0.268 , but it is more likely that the estimate is between -0.54 and -0.4 (Table 1). These observations lead to the conclusion that temperature in July and August is negatively associated to SSR prevalence.

Another example is the parameter of August precipitation (Fig. 3B). The posterior distribution and the corresponding quantiles given in Table 1 indicate that this parameter is most likely around 0 with a $2.5 \%$ probability of taking a value below -0.04 or a value above 0.058. Most of the mass of the posterior distribution $(99.4 \%)$ is in the positive and only $6 \%$ is in the negative space (Table 2). These observations lead to the conclusion that the effect of August precipitation on disease prevalence is rather positive but close to 0 . This observation agrees with the results of the logistic regression analysis, where we found that August precipitation was not significantly different from 0 at the $5 \%$ level of significance. Similar interpretations can be derived for all posterior parameter distributions (Tables 1 and 2).

Very informative prior distributions. Use of very informative prior distributions had an effect on the posterior distributions of the intercept, and the regression coefficients representing the effect of July and August precipitation, and the states of Iowa and

TABLE 1. Point estimates of logistic regression analysis and posterior distribution summaries of the parameters used to explain soybean Sclerotinia stem rot prevalence in the north-central region of the United States using noninformative prior distributions ${ }^{\mathrm{a}}$

\begin{tabular}{|c|c|c|c|c|c|c|c|}
\hline \multirow[b]{2}{*}{ Parameter } & \multirow{2}{*}{$\begin{array}{l}\text { Point estimates with } \\
\text { logistic regression }\end{array}$} & \multirow{2}{*}{$\begin{array}{c}\text { Mean of parameter } \\
\text { posterior distributions }\end{array}$} & \multirow{2}{*}{$\begin{array}{l}\text { Standard deviation of } \\
\text { posterior distributions }\end{array}$} & \multicolumn{4}{|c|}{ Quantiles of posterior distributions } \\
\hline & & & & $2.5 \%$ & $25 \%$ & $75 \%$ & $97.5 \%$ \\
\hline Intercept & 5.71 & 5.58 & 0.59 & 3.316 & 4.178 & 5.690 & 7.098 \\
\hline Average temperature of July and August & -0.4 & -0.47 & 0.103 & -0.678 & -0.543 & -0.404 & -0.268 \\
\hline July precipitation & 0.029 & 0.039 & 0.021 & -0.006 & 0.025 & 0.054 & 0.076 \\
\hline (No tillage) average temperature & -0.011 & -0.0104 & 0.022 & -0.054 & -0.025 & 0.004 & 0.031 \\
\hline (Minimum tillage) average temperature & 0.01 & 0.0137 & 0.0123 & -0.009 & 0.005 & 0.021 & 0.039 \\
\hline Iowa & 0.52 & 0.69 & 0.531 & -0.239 & 0.334 & 1.028 & 1.837 \\
\hline Minnesota & 0.98 & 1.181 & 0.532 & 0.216 & 0.821 & 1.519 & 2.284 \\
\hline Ohio & -0.82 & -1.09 & 0.945 & -3.178 & -1.678 & -0.405 & 0.52 \\
\hline August precipitation & - & 0.0104 & 0.025 & -0.04 & -0.006 & 0.029 & 0.058 \\
\hline
\end{tabular}

${ }^{a}$ Parameter posterior distribution summaries are based on Gibbs sampling for 8,000 to 10,000 iterations. 
Ohio (Table 3). These parameters exhibit "sensitivity to the choice of prior" distributions suggesting that the data do not contain overwhelming information about the effect of those factors on SSR prevalence. The most noticeable influence of the prior distri- butions on the parameter posterior distribution was observed on August precipitation and Ohio parameters.

The posterior distribution for August precipitation changed noticeably when a very informative prior distribution was used (Fig.

\section{Iteration}
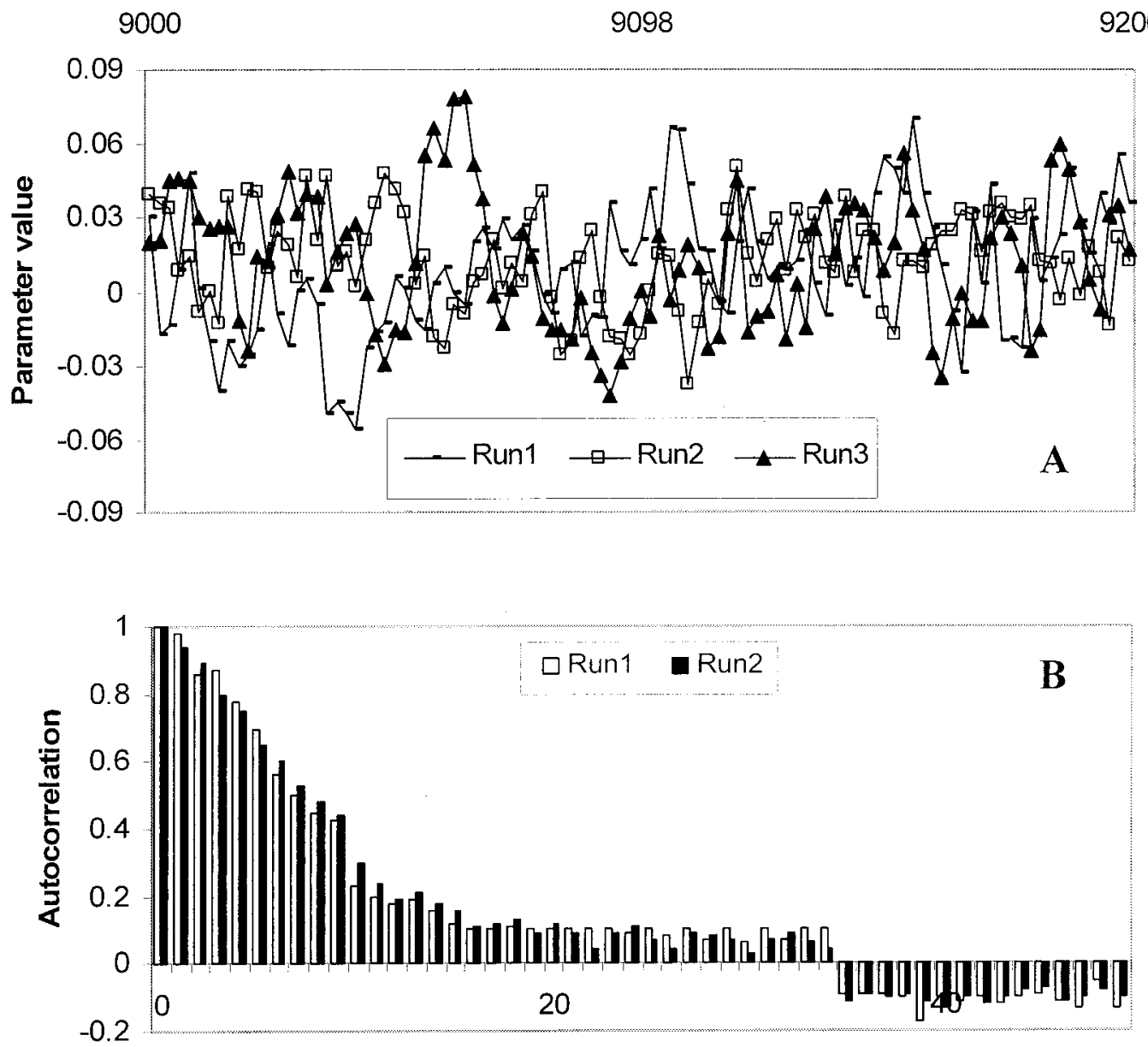

Lag

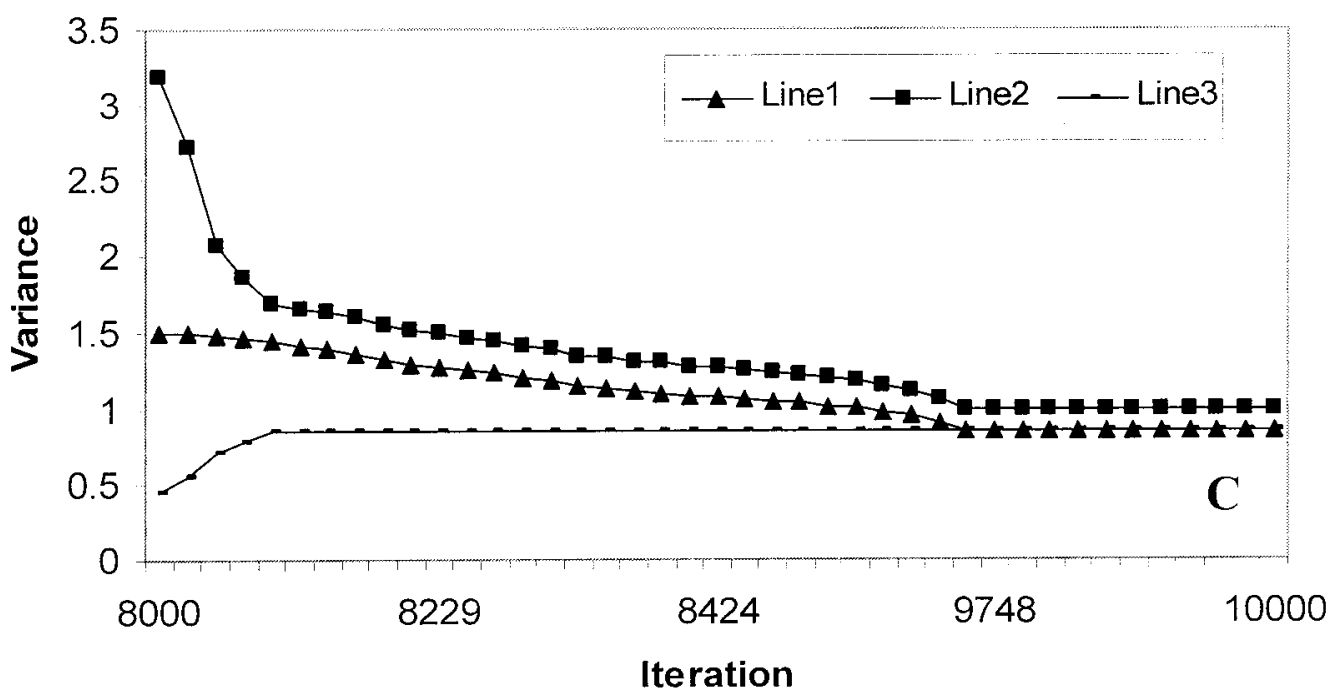

Fig. 2. A, Dynamic trace plot of no tillage indicator variable values against iteration number from three runs of the Gibbs sampler, B, plot of autocorrelation function of August precipitation variable out to lag 50, and $\mathbf{C}$, plot of the calculated Gelman-Rubin convergence statistic. Line 1 represents the width of the central $80 \%$ interval of the pooled runs, line 2 is the width of the $80 \%$ intervals within the individual runs, and line 3 is their ratio $(R=$ pooled/within). $R$ is expected to be approximately one in convergence. 
3D) in place of a noninformative prior distribution (Fig. 3B). With a very informative prior distribution, the posterior distribution for August precipitation was confined to the positive space (Fig. 3D) and the posterior mean shifted from 0.0104 (with noninformative prior distribution) to 0.1138 (with informative prior distribution).

The change in the posterior distribution of August precipitation indicates that the influence of the prior information on the form of the posterior distribution is nonnegligible. Thus, the choice of the prior distribution becomes important. Our assumptions for constructing the prior distribution were that the effect of precipitation during the month of August should be positive and about half of the July precipitation effect. If the sample data had been very informative for the parameter representing August precipitation, then the posterior distribution should look like the one obtained using a noninformative prior distribution. However, the posterior distribution changed considerably. This indicates that the analysis based on the sample data alone may not result in sufficiently accurate inference for the effect of precipitation during August on SSR prevalence.

The Ohio parameter has a negative posterior mean when a noninformative prior distribution is used, and a positive posterior mean with a very informative prior distribution (Tables 1 and 3). Furthermore, the posterior distribution quantiles and the probability that the effect of Ohio is negative or positive (Table 2) also change significantly when different priors are used. The August precipitation and Ohio parameters were not selected as statistically significant $(P=0.05)$ in the logistic regression analysis, where estimation was carried out using the method of maximum likelihood which relies exclusively on sample data (12).

\section{DISCUSSION}

We undertook the analysis of the SSR prevalence data from a Bayesian viewpoint to be able to derive a richer set of inferences about the effects of various factors on disease prevalence. The demonstrated sensitivity to the choice of prior distributions that was exhibited by some of the parameters in the model indicates that classical estimates, which are based solely on the sample information, should be viewed with caution. When noninformative priors were used, the means of the posterior distributions of each of the parameters closely approximated the point estimates obtained using the method of MLEs. This is to be expected, because a Bayesian analysis using noninformative priors is also based exclusively on the information that is provided by the sample data. When conducting the analysis from a Bayesian viewpoint, however, it is still possible to make a wider range of inferences about the model parameters and assess the uncertainty associated to the point estimates.

Incorporating information about parameter values into the analysis via the choice of informative prior distributions had a noticeable effect on the estimates (both point and distributional) that were obtained for some of the model parameters. As stated earlier, this is an indication that at least with respect to some factors, the sample data offers inconclusive evidence on their effect on SSR prevalence. Two factors stand out as particularly sensitive to whether extra-sample information is incorporated into the analysis or not: the effect of precipitation during the month of August and the effect of the state of Ohio.

Literature on SSR indicates that precipitation around the flowering stage $(9,13,15)$ can have a significant effect on disease occurrence. Most studies are focused on the effect of air temperature on sclerotia germination and apothecia production or SSR incidence and severity. The logistic regression analysis results (12) demonstrated that the absolute value of the average air temperature parameter was significantly higher than the value of the July total precipitation parameter $(0.4$ for average air temperature versus 0.029 for July precipitation), although both parameters were statistically significant $(P=0.05)$ for soybean SSR prevalence. August precipitation was not a statistically significant explanatory variable for the prevalence of SSR in the north-central

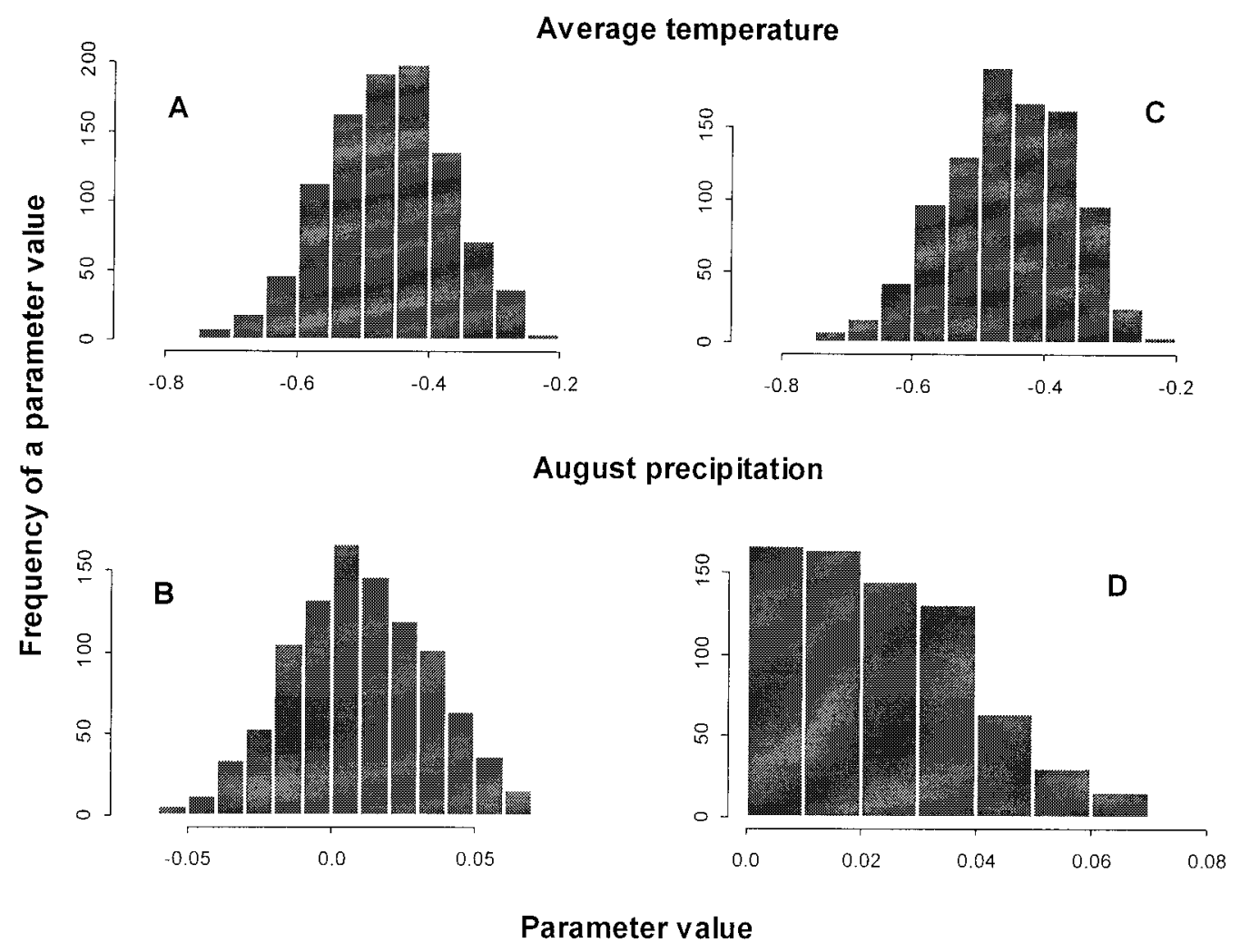

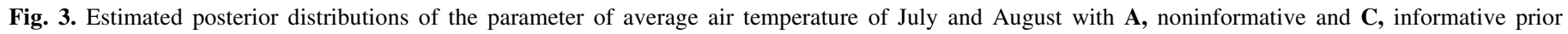
distributions and for the parameter of August precipitation with $\mathbf{B}$, noninformative and $\mathbf{D}$, informative prior distributions. 
region of the United States. In an earlier analysis (16), it was concluded that average temperature of July and August is a limiting factor for soybean SSR prevalence in the north-central region of the United States, whereas July and August precipitation is not a limiting factor.

Results of Bayesian analysis are not in conflict with these results but rather provide more insight on the various factors affecting SSR prevalence. For example, our analyses suggest that the 4-year data set is informative enough to give a reliable estimate of the effect of average air temperature during July and August on SSR prevalence but not for the effect of precipitation in July and August. During the 4 years of survey, precipitation in July and August were always at least as high as the 30-year average for the north-central United States. This may also explain the low parameter estimate for the effect of July and August precipitation that was obtained in logistic regression analysis (12).

Similar conclusions can be drawn for the state of Ohio. Only samples collected over 2 years from this state were available for analysis. Two years of data may not be enough to capture weather variability associated to location needed for explaining SSR prevalence in Ohio, especially if we consider that only four cases of SSR were observed in this state during that period. Thus, the sample size necessary for robust estimation of the regression coefficient associated to the Ohio indicator may need to be much larger than what was used in this study. This hypothesis appears credible if we take into account the fact that prevalence of SSR was low in Ohio.

If reliable extra-sample knowledge on the effect of the explanatory variables on the biological phenomenon exists, then Bayes' theorem provides the mechanism by which that information can be incorporated into the analysis. This prior knowledge may come from experts' opinions, published experimental results, or a combination of both. However, it is generally true that there is subjectivity in selecting the form of informative prior distributions to reflect that knowledge. Note that scientists who operate from within a classical framework also incorporate prior knowledge into experi- ment, but in a less formal way; in the classical paradigm, the design of an experiment is a vehicle for inclusion of prior information.

For Sclerotinia rot, literature is available on the factors affecting disease incidence or severity in several crops. Nevertheless, our focus on SSR prevalence and use of information on SSR incidence or severity to construct prior distributions for the parameters in the SSR prevalence model may not be appropriate. Incidence, severity, and prevalence are separate disease scales, and magnitude of the effect of explanatory variables may differ among these disease scales. Thus, we did not feel that enough extrasample information was available to construct very informative prior distributions for every factor in our model. Actually, the most informative prior distributions were imposed to July and August total precipitation. The choice of prior distributions for these parameters was arbitrary.

Although only a part of a more extensive analysis is presented here, several other informative prior distributions were considered, some of which did not have a clear biological interpretation but were suitable for examining the sensitivity of results to changes in the priors. It is noticeable that results were rather consistent across the wide range of prior assumptions; inferences of average air temperature of July and August were very stable, whereas those concerning the effect of July and August precipitation and Ohio State were sensitive to the choice of prior distributions. If the use of informative prior distributions improves the estimation of model parameters, then in addition, it will improve predictions of SSR prevalence in the north-central region of the United States. Prediction is a goal of modeling and is always associated with the data set used in model development. Prediction limitation due to data dependence is a topic of our current investigation and will be discussed in the future.

\section{APPENDIX}

Bayesian analysis was performed with an iterative, numerical approach called Gibbs sampler (GS). Implementation of the GS

TABLE 2. Probabilities of parameters used to explain soybean Sclerotinia stem rot prevalence in the north-central region of the United States to be negative or positive using noninformative and very informative prior distributions $\mathrm{s}^{\mathrm{a}}$

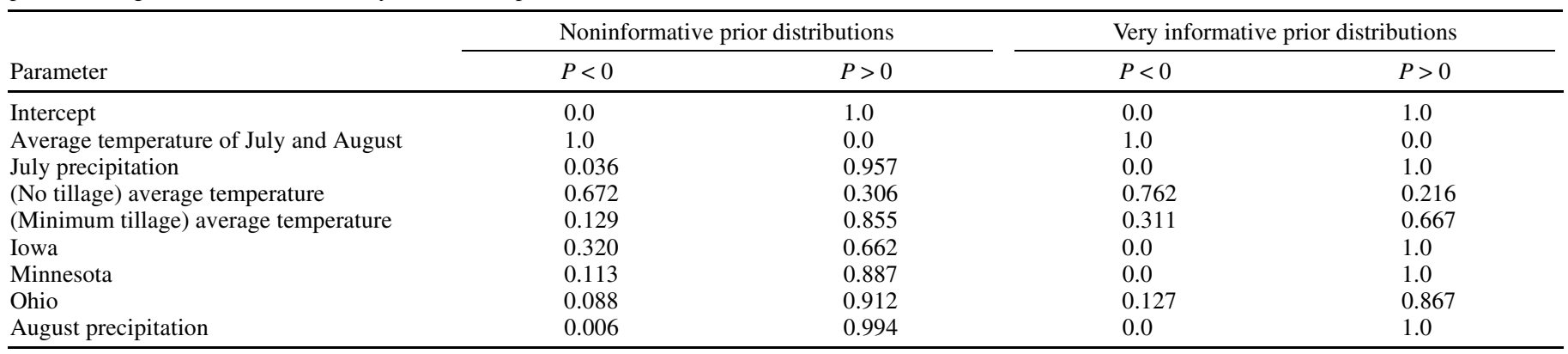

a Probabilities were calculated from parameter posterior distributions.

TABLE 3. Posterior distribution summaries of the parameters used to explain soybean Sclerotinia stem rot prevalence in the north-central region of the United States using very informative prior distributions ${ }^{\mathrm{a}}$

\begin{tabular}{|c|c|c|c|c|c|c|}
\hline \multirow[b]{2}{*}{ Parameter } & \multirow{2}{*}{$\begin{array}{c}\text { Mean of parameter } \\
\text { posterior distributions }\end{array}$} & \multirow{2}{*}{$\begin{array}{l}\text { Standard deviation of } \\
\text { posterior distributions }\end{array}$} & \multicolumn{4}{|c|}{ Quantiles of posterior distributions } \\
\hline & & & $2.5 \%$ & $25 \%$ & $75 \%$ & $97.5 \%$ \\
\hline Intercept & 2.86 & 0.528 & 1.983 & 2.765 & 3.098 & 4.990 \\
\hline Average temperature of July and August & -0.432 & 0.111 & -0.669 & -0.524 & -0.372 & -0.241 \\
\hline July precipitation & 0.1685 & 0.0173 & 0.133 & 0.157 & 0.18 & 0.203 \\
\hline (No tillage) average temperature & -0.0134 & 0.0187 & -0.05 & -0.026 & -0.001 & 0.024 \\
\hline (Minimum tillage) average temperature & 0.0052 & 0.0136 & -0.019 & -0.003 & 0.015 & 0.035 \\
\hline Iowa & 0.901 & 0.289 & 0.267 & 0.525 & 1.092 & 1.366 \\
\hline Minnesota & 1.353 & 0.43 & 0.431 & 0.796 & 1.638 & 2.042 \\
\hline Ohio & 0.044 & 0.0357 & -0.024 & 0.016 & 0.066 & 0.107 \\
\hline August precipitation & 0.1138 & 0.021 & 0.073 & 0.096 & 0.133 & 0.156 \\
\hline
\end{tabular}

${ }^{a}$ Parameter posterior distribution summaries are based on Gibbs sampling for 8,000 to 10,000 iterations. 
requires the specification of full conditional distributions of the parameters, i.e., the conditional distributions of each parameter given the values of all of the other parameters.

The response variable $Y_{i}$ is a binary variable taking the value 1 if any of the 20 stems in field $i$ was infected with Sclerotinia stem rot or 0 otherwise. Accordingly, the likelihood function has the Bernoulli form,

$$
Y_{i} \approx \operatorname{Bernoulli}\left(p_{i}\right)
$$

where the probability of infection $p_{i}$ was modeled as a function of weather, management practices, and field location. A logit transformation was used to linearize the association of $p_{i}$ and input variables,

$$
\begin{gathered}
\operatorname{logit}\left(p_{i}\right) \approx a_{0}+a_{1}(\text { average temp. })+a_{2}(\text { July prec. })+ \\
\left.\left.a_{3} \text { (tillage }\right)+a_{4}(\text { state })+a_{5} \text { (August prec. }\right)
\end{gathered}
$$

where $a_{0} \approx$ noninformative, $a_{1} \approx$ noninformative/informative, $a_{2} \approx$ noninformative/very informative, $a_{3} \approx$ noninformative/very informative, $a_{4} \approx$ noninformative/very informative, and $a_{5} \approx$ noninformative/very informative.

\section{LITERATURE CITED}

1. Abawi, G. S., and Grogan, R. G. 1975. Source of primary inoculum and effects of temperature and moisture on infection of beans by Whetzelinia sclerotiorum. Phytopathology 65:300-309.

2. Abawi, G. S., and Grogan, R. G. 1979. Epidemiology of diseases caused by Sclerotinia species. Phytopathology 69:899-904.

3. Boland, G. J., and Hall, R. 1988. Epidemiology of Sclerotinia stem rot of soybean in Ontario. Phytopathology 78:1241-1245.
4. Dunson, D. B. 2001. Commentary: Practical advantages of Bayesian analysis of epidemiological data. Am. J. Epidemiol. 153:1222-1226.

5. Garcia-Garza, J. A., Boland, G. J., and Vyn, T. J. 1998. Influence of crop rotation and reduced tillage on white mold of soybean caused by Sclerotinia sclerotiorum. (Abstr.) Phytopathology 88:533.

6. Gelman, A. 1996. Inference and Monitoring Convergence in Markov Chain Monte Carlo in Practice. W. R. Gilks, S. Richardson, and D. J. Spiegelhalter, eds. Chapman \& Hall, New York.

7. Gelman, A., Carlin, J. B., Stern, H. S., and Rubin, D. B. 1996. Bayesian Data Analysis. Chapman \& Hall, New York.

8. Grau, C. R. 1989. Sclerotinia stem rot. Pages 47-48 in: Compendium of Soybean Diseases. 3rd ed. J. B. Sinclair and P. A. Backman, eds. The American Phytopathological Society, St. Paul, MN.

9. Haas, J. H., and Bolwyn, B. 1972. Ecology and epidemiology of Sclerotinia wilt of white beans in Ontario. Can. J. Plant Sci. 52:525-533.

10. Kurle, J. E., Grau, C. R., Oplinger, E. S., and Mengistu, A. 2001. Tillage, crop sequence, and cultivar effects on Sclerotinia stem rot incidence and yield in soybean. Agron. J. 93:973-982.

11. Lawson, A. B. 2001. Tutorial in biostatistics: Disease map reconstruction. Stat. Medic. 20:2183-2204.

12. Mila, A. L., Yang, X. B., Carriquiry, A. L., and Zhao, J. 2001. Modeling the prevalence of soybean Sclerotinia stem rot and the associated economic outcomes in the North Central region of USA. Proc. Int. Workshop Plant Epidemiol., 8th. Braz. Phytopathol. Soc., Ouro Peeto, Brazil.

13. Moore, W. D. 1955. Relation of rainfall and temperatures to the incidence of Sclerotinia sclerotiorum in vegetables in south Florida during the years 1944 to 1954. Plant Dis. Rep. 39:470-471.

14. Natti, J. J. 1971. Epidemiology and control of bean white mold. Phytopathology 61:669-674.

15. Twengstrom, E., Sigvald, R., Svensson, C., and Yuen, J. 1998. Forecasting Sclerotinia stem rot in spring sown oilseed rape. Crop Prot. 17:405-411.

16. Workneh, F., and Yang, X. B. 2000. Prevalence of Sclerotinia stem rot of soybean in north-central United States in relation to tillage, climate, and latitudinal positions. Phytopathology 90:1375-1382. 